\title{
An Experimental Study of Parameter Selection in Particle Swarm Optimization Using an Automated Methodology
}

\author{
María Cosío-León ${ }^{1}$, Anabel Martínez-Vargas ${ }^{2}$, and Everardo Gutierrez ${ }^{3}$ \\ 1 Universidad Autónoma de Baja California, FIAD, Ensenada, BC, Mexico \\ ${ }^{2}$ Centro de Investigación y Desarrollo de Tecnología Digital del Instituto Politécnico \\ Nacional (CITEDI-IPN), Tijuana, BC, Mexico \\ 3 Universidad Autónoma de Baja California, FC, Ensenada, BC, Mexico \\ cosio.maria@uabc.edu.mx, amartinez@citedi.mx, \\ everardo.gutierrez@uabc.edu.mx
}

\begin{abstract}
In this work, an experimental study to evaluate the parameter vector utility brought by an automated tuning tool, so called Hybrid Automatized Tuning procedure (HATp) is given. The experimental work uses the inertia weight and number of iterations from the algorithm PSO; it compares those parameters from tuning by analogy and empirical studies. The task of PSO is to select users to exploit concurrently a channel as long as they achieve the Signal-to-Interference-Ratio (SINR) constraints to maximize throughput; however, as the number of users increases the interference also arises; making more challenging for PSO to converge or to find a solution. Results show that, HATp is not only able to provide a parameter vector that improve the search ability of PSO to find a solution but also to enhance its performance on resolving the spectrum sharing application problem than those parameters values suggested by empirical and analogical methodologies in the literature on some problem instances.
\end{abstract}

Keywords: Parameter tuning, metaheuristic, particle swarm optimization.

\section{Introduction}

Meta-heuristic algorithms are black box procedures that, provided a set of candidate solutions, solve a problem or a set of problems instances. However, they require to select a set of parameters to tuning them, which greatly affect the meta-heuristic's efficiency to solve a given decision problem. Those parameters are classified as qualitative and quantitative; the former are related to procedures (e.g Binary or Continuous PSO), while the latter are associated with specific values (e.g. number of iterations, and population size). This work is focused on quantitative parameters to configure the PSO algorithm; which is a non-trivial problem as authors in [1] explain. This problem in the literature is called algorithm configuration by authors in [2]; and parameter tuning in [1], [3]. 
In [1] authors define the parameter tuning procedure as the task in which parameter values are set before executing a given meta-heuristic; and those values remain fixed while the meta-heuristic is running. Due to the aforementioned, parameter's tuning is an important task in the context of developing, evaluating and applying meta-heuristic algorithms.

\subsection{Particle Swarm Optimization Algorithm}

To evaluate parameter vector utility bring by the tuning procedure, this paper uses the Particle Swarm Optimization (PSO) algorithm [4], which is categorized by its authors as an evolutionary computation technique since it utilizes a population of candidate solutions to evolve an optimal or near-optimal solution for a problem.

The individuals in the PSO technique are called particles and they represent a possible solution of the optimization problem. When elements of a problem are represented as binary variables, the binary version of PSO (BPSO) is used [7]. Since its inception, many adjustments have been made to improve its performance. One of these new improvements to BPSO algorithms is Socio-Cognitive Particle Swarm Optimization (SCPSO) [8]. SCPSO introduces the distance between gbest and pbest values as a new velocity update equation which maintain diversity in the swarm, a socio-cognitive scaling parameter $c_{3}$ and a new position update equation. The latter used on spectrum sharing application to maximize throughput in the network.

This feature article is about analyzing two procedures for optimization parameters on SCPSO algorithm: a) model-base CALIBRA algorithm [9], and b) polynomial interpolation technique called Newton's Divided Difference Polynomial Method of Interpolation [10]. Along with aforementioned procedures, we use as a control group, parameter vector values taken from the state of art, tuning by analogy (TA) and empirical methodology to test the parameter vector utility.

\section{Automatic Parameter Tuning}

The automated tuning procedures address the parameter tuning problem; they are designed to search for the best parameter vector. Therefore, given a metaheuristic with $n$ parameters, tuning procedures search for the best parameter vector $P^{*}=\left\{p_{0}, p_{1}, \ldots, p_{n}\right\}$. The parameter vector $P^{*}$ usually is selected by researchers using manual tuning procedures [11] or tuned by analogy's procedures [12]. The No Free Lunch theorem of optimization states that; one $P^{*}$ allowing to solve all optimization problems is verifiable non-existent; therefore, tuning by analogy procedure, which uses a single parameter vector for different problems or different problem instances, is not the best strategy. On the other hand, manual tuning procedures are very time consuming, and failure prone; therefore, it is necessary to conduct other procedures to avoid those drawbacks. 
In [13] the author gives a brief review about the automated parameter tuning procedures; using a two fold model classification: a) model-free, and b) modelbased approaches. The former models are procedures guided by randomness, or simple experimental design (e.g. Latin Hypercube Sampling), tuners with very limited extrapolation potential. On the other hand, the latter models have the capabilities of 1) interpolating for the choice of new parameter settings; and even 2) extrapolating parameter vectors for new problems or problem instances. In this context, interesting contributions to find $P^{*}$ through automated procedures are presented in [1], [3].

In the next section, we will describe CALIBRA, and Newton's Divided Difference Polynomial Method of Interpolation which is the the interpolation technique selected to find new $P^{*}$ for problem instances.

\subsection{The Hybrid Automatized Tuning Procedure (HATp)}

Traditional tuning methods comprises three layers: a) design layer; b) algorithm layer; and c) application layer [1]. In this experimental study, we propose to use in the design layer an Hybrid Automatized Tuning procedure (HATp). Firstly, it exploits a procedure that couples fractional factorial experimental design and a local search procedure, called CALIBRA [9]. Then, an interpolation method such as Newton's divided difference polynomial works with CALIBRA to bring a particular $P^{*}$ for problem instances; while reducing computer time.

HATp's first stage uses CALIBRA (HATpI); it sets up a series of experiments to find the best value for quantitative parameters in the tuning target algorithm. The notion of best depends on how the performance of the target algorithm is measured. To achieve this, CALIBRA combines two methods: experimental designs and local search. The experimental designs focus on the on promising regions [9]. Promising regions are selected using a full factorial design $2^{k}$, and Taguchi's $L_{9}\left(3^{4}\right)$; once a region is selected, CALIBRA makes a local search. The above procedure is executed until certain stopping condition is met. CALIBRA uses $P$ to configure the interest algorithm; same process is executed several times with $P$ obtained from promising regions by CALIBRA up to find $P^{*}$. It is important to denote that the CALIBRA software can provided up to five parameter calibration; so for metaheuristics with more than five parameters, it is necessary to develop a new CALIBRA software version.

Considering the No Free Lunch theorem of optimization; and a continuous local function $f(x)$; once CALIBRA brought a set of parameter vectors $P^{*}$, HATp uses a polynomial interpolation method to find new problem instances $P^{*}$ (HATpII). The interpolation process takes advantage of CALIBRA model-base characteristic; building a polynomial of order $n$ that passes through the $1+n$ points calculated by CALIBRA. To find the new points, the interpolation process uses Newton's divided differences recursive equations (1), (2), (3):

$$
\begin{aligned}
f\left[x_{i}\right] & =y_{i}=f\left(x_{i}\right) \\
f\left[x_{i}, x_{i+1}\right] & =\frac{f\left[x_{i+1}\right]-f\left[x_{i}\right]}{x_{i+1}-x_{i}}
\end{aligned}
$$


María Cosío-León, Anabel Martínez-Vargas, and Everardo Gutierrez

$$
f\left[x_{i+1}, x_{i+2}, \ldots, x_{i+n}\right]=\frac{f\left[x_{i+1}, x_{i+2}, \ldots, x_{i+n}\right]-f\left[x_{i}, x_{i+1}, x_{i+2}, \ldots, x_{i+n-1}\right]}{x_{i+n}-x_{i}}
$$

Formalizing the automated parameter tuning procedure HATp: suppose that the performance of algorithm $A c$ is to be studied for a given set of problem instances $I ; P^{*}$ is found using a model based algorithm $C a$; using a set of problems instances $I^{\prime}$ different to $I$. Once $C a$ brings the $P^{*}$, the algorithm $A c$ is configured with it, and a problem instance from $I$ is resolved. Performance measures are selected according to problem instances open questions.

The second strategy in HATp is an interpolation procedure, $D d$ to find $P^{*}$ for new problem instances as follows: given a continuous function $f$ and a sequence of known points $x_{0} ; x_{1} ; \ldots ; x_{n}$. the divided difference of $f$ over $x_{0} ; x_{1} ; \ldots ; x_{n}$ points is the value of $a_{n}=f\left[x_{0} ; x_{1} ; \ldots ; x_{n}\right]$; which is recursively computed by equations $(1),(2),(3)$, in intention to find $P^{*}$, and reduce tuning computer time.

\section{Target Problem and Experimental PSO Setup}

In cognitive wireless networks with spectrum underlay when a secondary transmitter requests for a primary channel, they must be able to check if mutual interference among secondary users (unlicensed users) and primary users (licensed users) doesn't rise to the level of harmful interference. In this case the primary users have priority over a specific channel, and secondary users are allowed to transmit in the same channel as long as they do not cause harmful interference to the primary user.

Consider Figure 1, there is a number of secondary links $S l$ and primary links $P l$ are deployed in a coverage area $A$. A link either secondary or primary is represented by the union of a transmitter and a receiver and it is identified by a number beside the link. The number of primary links $P l$ is the primary network, which is assigned with a portion of regulated spectrum. Whereas, the secondary network is composed by the number of secondary links $S l$, which have to find a primary channel to exploit it. The cognitive network has a central entity; it knows the number of primary channels that can be assigned to secondary links. The primary channel allocation for secondary links doesn't depend on whether primary channels are idle or busy but once they are assigned the interference does not cause disruption in both primary and secondary networks. A primary link has a primary channel to share (the numbers in braces in Figure 1) and one primary channel can be assigned to several secondary links (the number in brackets in Figure 1), as long as they, together, do not generate enough interference to disrupt the primary communication link. The secondary link selection depends on how much interference it can generate to those primary and secondary links that use the same primary channel. To determine the level of interference that any of the links experiences in the cognitive network, the equations (4) and (5) calculate the signal-to-interference-noise-ratio (SINR) value that the receiver either secondary or primary can suffer. The SINR at the secondary receiver $u$ is 


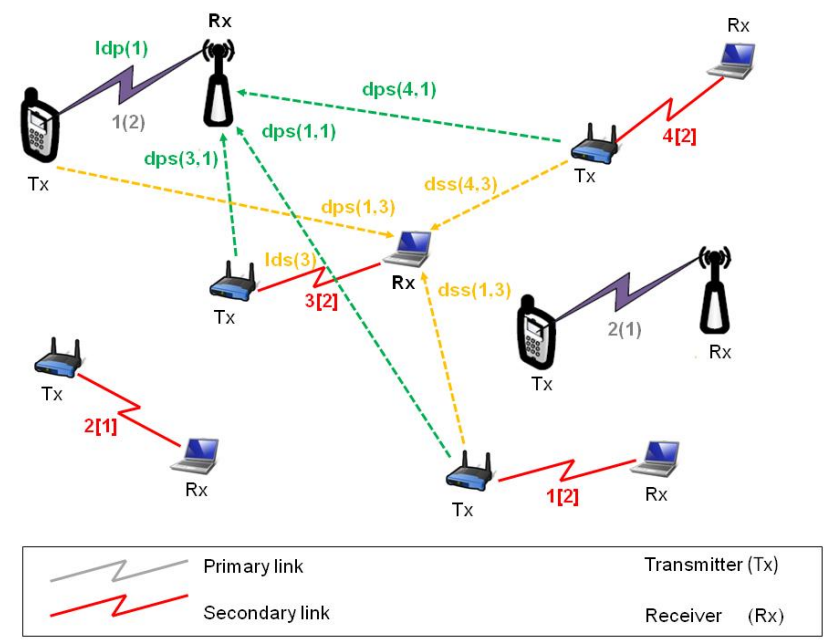

Fig. 1. System scenario.

given by:

$$
S I N R_{u}=\frac{P_{u} / l d s(u)^{n}}{\sum_{k \in \Phi} P_{k} / d s s(k, u)^{n}+P_{v} / d p s(v, u)^{n}}, 1 \leq u \leq S l
$$

where $P_{u}$ is the transmit power of secondary transmitter $u, P_{k}$ is the transmit power of secondary transmitter $k, P_{v}$ is the transmit power of primary transmitter $v, l d s(u)$ is the link distance of secondary link $u, d s s(k, u)$ is the distance from secondary transmitter $k$ to secondary receiver $u, \operatorname{dps}(v, u)$ is the distance from primary transmitter $v$ to secondary receiver $u, k$ is the index of active secondary transmitters, $\Phi$ is the set of active secondary transmitters, $n$ is the path loss exponent (a value between 2 and 4). Similarly, the SINR at the primary receiver $v$ is given by:

$$
S I N R_{v}=\frac{P_{v} / l p d(v)^{n}}{\sum_{k \in \Phi} P_{k} / d p s(k, v)^{n}}, 1 \leq v \leq P l
$$

where $P_{v}$ is the transmit power of primary transmitter $v, P_{k}$ is the transmit power of secondary transmitter $k, \operatorname{ldp}(v)$ is the link distance of primary link $v$, $d p s(k, v)$ is the distance from secondary transmitter $k$ to primary receiver $v$.

Data rate contributions of the secondary links and primary links are calculated according to equations (6) and (7) respectively. The data rate depends on primary channel bandwidth $B$ that secondary links and primary links can share and the conditions of the propagation environment (attenuation and interference).

$$
\begin{aligned}
c_{u}^{\prime} & =B \log _{2}\left(1+S I N R_{u}\right) \\
c_{v}^{\prime \prime} & =B \log _{2}\left(1+S I N R_{v}\right)
\end{aligned}
$$


Based on the above discussion, the admission and interference control problem is formulated as the following optimization problem:

$$
\operatorname{Max} \sum_{u=1}^{S l} c_{u}^{\prime} x_{u}+\sum_{v=1}^{P l} c_{v}^{\prime \prime}
$$

s.t.

$$
\begin{aligned}
& S I N R_{u} \geq \alpha \\
& S I N R_{v} \geq \beta \\
& c_{u}^{\prime}>0, \quad u=1,2, \ldots, S l \\
& c_{v}^{\prime \prime}>0, \quad u=1,2, \ldots, P l \\
& c_{u}^{\prime}, c_{v}^{\prime \prime} \in R^{+} \\
& x_{u}=\left\{\begin{array}{l}
1, \text { if } S I N R_{u} \geq \alpha \text { and } S I N R_{v} \geq \beta \\
0, \text { otherwise }
\end{array}\right.
\end{aligned}
$$

By observing the above optimization problem, the objective function is to maximize the sum throughput in the cognitive network (8), subject to the SINR requirements of the secondary links (9) and primary links (10). The maximum interference level is limited by $\alpha$ in the secondary network and $\beta$ in the primary network in the right-hand side of each of the constraints (9) and (10). Constraints from (11) to (13) are integrity restrictions. $x_{u}=1$ if secondary link $u$ is included in the solution and $x_{u}=0$ if it remains out as indicated in (14).

\subsection{Solution Procedure Based on SCPSO Algorithm}

The goal by using SCPSO is to decide which secondary links can achieve this, finding a binary vector $P_{g}$ of size $S l$ representing the solution, where the bits $1 / 0$ symbolize if the $u-t h$ secondary link is selected as part of the solution (bit 1 ) or not (bit 0). The maximum data rate achieved in the system is $f\left(P_{g}\right)$.

Assume $S$ as the number of particles and $D$ as the dimension of particles. A candidate solution is expressed as $X_{i}=\left[x_{i 1}, x_{i 2}, \ldots, x_{i D}\right]$ where $x_{i d} \in\{0,1\}$. Velocity is $V_{i}=\left[v_{i 1}, v_{i 2}, \ldots, v_{i D}\right]$ where $v_{i d} \in\left[-V_{\max }, V_{\max }\right]$. The personal best evaluation (pbest) of the $\mathrm{i}$-th particle is denoted as $P_{i}=\left[p_{i 1}, p_{i 2}, \ldots, p_{i D}\right]$ where $p_{i d} \in\{0,1\} . g$ is the index of the best particle in the swarm, therefore $P_{g}$ is the best evaluation in the swarm (gbest). The swarm is manipulated according to the following velocity $v_{i d}$ and position $x_{i d}$ equations:

$$
\begin{aligned}
v_{i d} & =w v_{i d}+c_{1} r_{1}\left(p_{i d}-x_{i d}\right)+c_{2} r_{2}\left(p_{g d}-x_{i d}\right) \\
v_{i d} & =w^{1} v_{i d}+c_{3}(\text { gbest }- \text { pbest }) \\
x_{i d} & =x_{i d}+v_{i d} \\
x_{i d} & =x_{i d} \bmod (2)
\end{aligned}
$$

where $w$ and $w^{1}$ are considered the inertia weights, $c_{1}$ and $c_{2}$ are the learning factors, $c_{3}$ is called as socio-cognitive scaling parameter, and finally $r_{1}$ and $r_{2}$ 
An Experimental Study of Parameter Selection in Particle Swarm Optimization ...

are uniformly distributed random numbers in [0,1]. Algorithm 1 is a simplified version from work presented in [14] to address the spectrum underlay problem in cognitive networks.

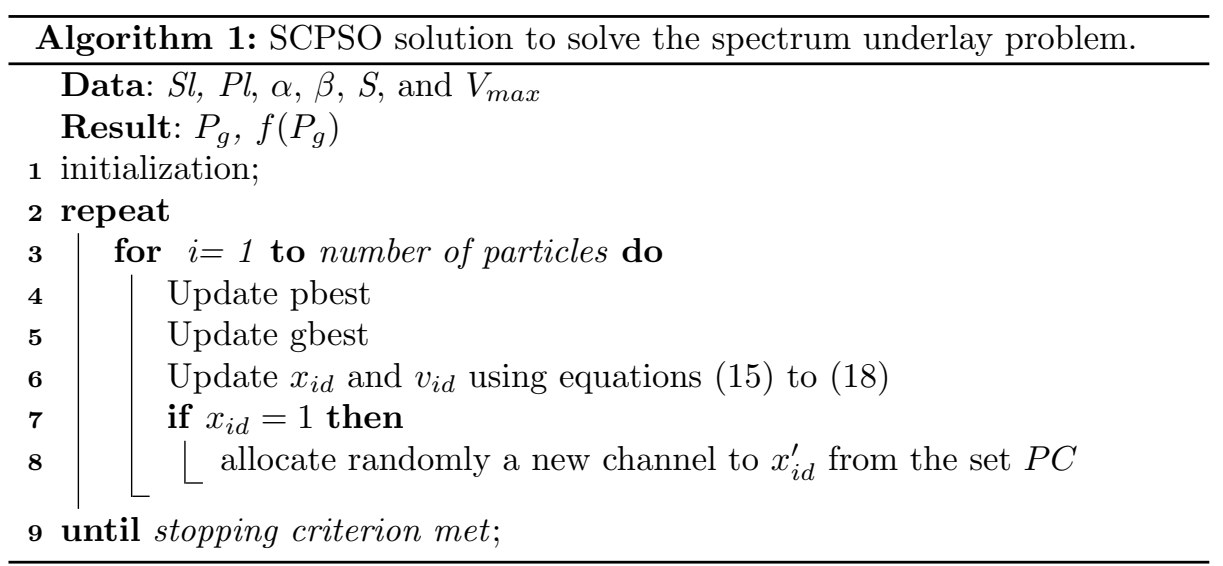

Initialization stage includes: 1) locate randomly $S l$ and $P l$ in the scenario, 2) initialize randomly $\left.X_{i}, 3\right)$ initialize randomly $V_{i}$, 4) Set $\left.P_{i}=X_{i}, 5\right)$ Set $P_{i}^{\prime}=X_{i}^{\prime}$, and 6) initialize randomly vector Spectrum Status with values from $P l$. Note that in initialization stage, $P_{i}$ and $X_{i}$ are considered to coincide. Three new vectors $X_{i}^{\prime}, P_{i}^{\prime}$, and Spectrum Status are included additionally. $X_{i}^{\prime}$ provides the possible channel allocation for secondary links. $P_{i}^{\prime}$ stores the best channels allocations found so far for a particle and Spectrum Status vector stores the channel allocations for primary links.

In update pbest (step 4 in Algorithm 1), the particle compares $f\left(X_{i}\right)>f\left(P_{i}\right)$ and overwrites pbest if $f\left(X_{i}\right)$ is higher than $f\left(P_{i}\right)$. In contrast, in update gbest, all pbest values will be compared with gbest value, so if there is a pbest which is higher than the gbest, then gbest will be overwritten. Update pbest and gbest phases require fitness calculation according to (8); to avoid infeasible solutions in the swarm, they are penalized by setting total particle's fitness to zero therefore they are not chosen in the selection process. Further details and the complete implementation of this solution procedure based on the SCPSO algorithm are provided in [14].

\subsection{Quantitative Parameters: Number of Iterations and Inertia Weight}

The SCPSO parameters of interest in this paper are the number of iterations and inertia weight. The inertia weight $w$ influences the trade-off between exploration and exploitation [15]; therefore, a large $w$ facilitates exploration, while a smaller $w$ tends to facilitate exploitation in promising regions. Finding a suitable $w$ helps to require fewer number of iterations on average to find the optimum value [15]. We took the reference values suggested by analogy from [8], except for the number of iterations and swarm size which are derived from an 
empirical tuning methodology (see Table 1); those values and $H A T p P^{*}$ were tested in the SCPSO algorithm to know their utility; it is important to denote that both parameter vectors had same values for parameters indicated $\left(^{*}\right)$ in Table 1.

Table 1. Parameter values.

\begin{tabular}{lr}
\hline Parameter & Value \\
\hline Number of Secondary Users $\left(^{*}\right)$ & $15,20,25$ and 30 \\
Number of Primary Users $\left(^{*}\right)$ & 1 \\
Number of Particles $\left(^{*}\right)$ & 40 \\
Number of iterations & 150 \\
Maximum velocity $\left(^{*}\right)$ & 6 \\
Minimal Velocity $\left(^{*}\right)$ & -6 \\
Inertia Weight & 0.721000 \\
\hline
\end{tabular}

Taking as pivotal values, the Number of iterations and Inertia weight showed in Table 1; we define a 200 hundred percent rule to state thresholds around them. It is a precondition in CALIBRA to define a searching area for promise regions.

Using aforementioned thresholds, CALIBRA defines a set of $P$ vectors which are used to configure the set $I^{\prime}$ of problem instances; finally after testing $P$ vectors on each problem instance in $I^{\prime}$; CALIBRA brought a $P^{*}$. The combination of $\alpha, \beta$ and the Number of secondary users is used by CALIBRA to find $P^{*}$. Note that $\alpha$ and $\beta$ are considered to coincide. Tables 2 and 3 show the entire design points used to configure SCPSO algorithm to resolve the set of problem instances $I$.

Table 2. Number of iteration values brought by CALIBRA.

\begin{tabular}{|c|c|c|c|c|c|}
\hline \multirow{2}{*}{\multicolumn{2}{|c|}{$\begin{array}{l}\text { Number of } \\
\text { Iterations }\end{array}$}} & \multicolumn{4}{|c|}{ Number of Secondary Users } \\
\hline & & 15 & 20 & 25 & 30 \\
\hline \multirow{6}{*}{$\begin{array}{r}\alpha, \beta \\
(\mathrm{dB})\end{array}$} & 4 & 48 & 198 & 168 & 162 \\
\hline & 6 & 228 & 102 & 128 & 142 \\
\hline & 8 & 96 & 93 & 145 & 227 \\
\hline & 10 & 122 & 222 & 221 & 246 \\
\hline & 12 & 31 & 201 & 199 & 14 \\
\hline & 14 & 145 & 197 & 258 & 82 \\
\hline
\end{tabular}

The range of $w$ values brough by CALIBRA contained values gave in [8] $w=0.721000$, and [15] $w=0.8$ as show in Table 3 . On the other hand, the number of iterations have differences, in [15] authors proposed up to 2500 iterations, the empirical tuning result was 150 , and the values brough by CALIBRA between 40 and 250 for the number of iterations (see Table 2). 
An Experimental Study of Parameter Selection in Particle Swarm Optimization ...

Table 3. Inertia weight values brought by CALIBRA.

\begin{tabular}{|c|c|c|c|c|c|}
\hline \multirow{2}{*}{ Inertia weight } & \multicolumn{4}{|c|}{ Number of Secondary Users } \\
\cline { 3 - 6 } \multicolumn{2}{|c|}{} & 15 & 20 & 25 & 30 \\
\hline \multirow{4}{*}{$\alpha, \beta$} & 4 & 0.72309 & 0.81257 & 0.79076 & 0.78843 \\
$(\mathrm{~dB})$ & 6 & 0.62500 & 0.82340 & 0.88750 & 0.70429 \\
& 8 & 0.86409 & 0.76332 & 0.95552 & 0.93976 \\
& 10 & 0.85324 & 0.88784 & 0.89460 & 0.80000 \\
& 12 & 0.71726 & 0.80693 & 0.80002 & $\mathbf{0 . 1 1 0 9 3}$ \\
& 14 & 0.88921 & 0.77942 & 0.94360 & $\mathbf{0 . 4 5 0 0 0}$ \\
\hline
\end{tabular}

\section{Results}

The aim of this experimental study is to know how much the SCPSO algorithm performance is affected by $P^{*}$ brought by TA-empirical tuning and HATp processes. Tables 4 and 5 show SCPSO algorithm results using 30 different design points defined by parameter values in Tables 2 and 3; those design points were tested 1000 times; The characteristics of the computer equipment and software used were: a)Fine Tuner Tool, Calibra; Language, Borland C++, version 5.02; Operating system, Windows 7 enterprise 32 bits; Processor, Intel(R) Core (TM) i5-2320 CPU@3.00 GHz, and the RAM memory, 4.00 GB.

Analysing the SCPSO mean throughput in Table 5; it was higher when the SCPSO algorithm used the TA-empirical tuning vector than HATpI; however, as the number of secondary users, $\alpha, \beta$ values increase, also increases the average throughput of the SCPSO using the HATpI $P^{*}$, up to $100 \%$. Concluding, the TA-empirical $P^{*}$ utility is better with low problem complexity, while HATpI $P^{*}$ is better in scenarios with high problem complexity. In line 48 of Table 5 HATpI $P^{*}$ had its worse performance, when the number of secondary users is eqauls to 30 and $\alpha, \beta=14 \mathrm{~dB}$, the highest problem instance complexity; due to fact that CALIBRA did not provide a $P^{*}$. About the maximum value for data rate, as problem complexity increase the utility of HATpI $P^{*}$ as well. However the median parameter shows zero in both process.

The SCPSO algorithm performance in Table 4 is similar to the one shown in Table 5. Although, considering the average throughput, only in three cases the $T A$ vector allowed SCPSO algorithm to bring better results.

A global view of results in Tables 4 and 5, show that as the problem complexity increases, the SCPSO algorithm performance degrades. This behaviour allow us to conclude that, taking higher thresholds for $w$ and Number of iterations could be possible to find better $P^{*}$ vectors. This conclusion is supported by [8] and [15] as well as CALIBRA exploration in similar areas, having SCPSO low performance on average fitness for entire problem instances.

\section{Conclusions}

In this paper, we analyse two parameter tuning procedures, specifically focusing on two quantitative parameters of SCPSO which resolves the spectrum sharing 
María Cosío-León, Anabel Martínez-Vargas, and Everardo Gutierrez

Table 4. Tuning by analogy versus Interpolation $P_{i}^{*}$ SCPSO results.

\begin{tabular}{|c|c|c|c|c|c|c|c|}
\hline & Design Point & Mean & $\begin{array}{l}\text { Standard } \\
\text { Deviation }\end{array}$ & Q1 & Median & Q3 & Maximum \\
\hline 1 & $4-17$ & 696.8936 & 216.1854 & 561.3409 & 685.2271 & 825.2401 & 1579.2489 \\
\hline 2 & 4-17-HATpII & 705.1499 & 190.4909 & 580.8800 & 697.3810 & 820.5509 & 1336.9885 \\
\hline 3 & $6-17$ & 617.4922 & 216.0871 & 507.0424 & 624.5074 & 755.0196 & 1360.8872 \\
\hline 4 & 6-17-HATpII & 622.5656 & 223.6225 & 494.9839 & 626.1765 & 761.4327 & 1330.2744 \\
\hline 5 & $8-17$ & 536.3193 & 259.6028 & 409.4786 & 551.6591 & 700.4633 & 1298.0047 \\
\hline 6 & 8-17-HATpII & 559.0188 & 229.8708 & 429.6519 & 568.1481 & 700.5008 & 1452.1818 \\
\hline 7 & $10-17$ & 395.5316 & 266.3749 & 200.4630 & 441.9605 & 580.0636 & 1288.5817 \\
\hline 8 & 10-17-НАTpII & 530.5890 & 188.5329 & 412.0709 & 525.8743 & 651.5861 & 1333.6288 \\
\hline 9 & $12-17$ & 243.9879 & 255.2588 & 0 & 244.8208 & 450.1429 & 950.3957 \\
\hline 10 & 12-17-HATpII & 168.5188 & 209.0008 & 0 & 0 & 330.8397 & 774.7756 \\
\hline 11 & $14-17$ & 135.0718 & 199.5533 & 0 & 0 & 288.34135 & 863.5317 \\
\hline 12 & 14-17-НATpII & 168.5188 & 209.0008 & 0 & 0 & 330.83975 & 774.7756 \\
\hline 13 & $4-22$ & 577.0828 & 314.40666 & 438.41045 & 625.3864 & 784.41305 & 1502.3393 \\
\hline 14 & 4-22-HATpII & 665.16352 & 238.7602 & 538.10245 & 666.6439 & 807.373 & 1390.4193 \\
\hline 15 & $6-22$ & 424.30708 & 340.36649 & 0 & 508.0161 & 683.1996 & 1557.0939 \\
\hline 16 & 6-22-HATpII & 579.12143 & 267.8317 & 452.0497 & 594.8837 & 743.12315 & 1527.0990 \\
\hline 17 & $8-22$ & 253.58628 & 319.7857 & 0 & 0 & 542.4337 & 1368.1840 \\
\hline 18 & 8-22-HATpII & 428.06549 & 308.7124 & 0 & 497.0066 & 651.2654 & 1354.8228 \\
\hline 19 & $10-22$ & 121.20538 & 235.4958 & 0 & 0 & 0 & 1107.1568 \\
\hline 20 & 10-22-HATpII & 404.11412 & 275.4165 & 191.6255 & 445.3831 & 598.9975 & 1116.7342 \\
\hline 21 & $12-22$ & 43.86616 & 140.1225 & 0 & 0 & 0 & 717.4726 \\
\hline 22 & 12-22-НАТрII & 197.78717 & 258.8344 & 0 & 0 & 413.1131 & 1072.9170 \\
\hline 23 & $14-22$ & 17.44797 & 84.5748 & 0 & 0 & 0 & 806.7283 \\
\hline 24 & 14-22-HATpII & 98.76469 & 189.0751 & 0 & 0 & 0 & 792.4581 \\
\hline 25 & $4-27$ & 257.6821 & 354.0673 & 0 & 0 & 604.3964 & 1226.8559 \\
\hline 26 & 4-27-HATpII & 454.0794 & 375.7800 & 0 & 543.2218 & 751.57415 & 1407.41 \\
\hline 27 & $6-27$ & 124.4342 & 276.2780 & 0 & 0 & 0 & 1327.9231 \\
\hline 28 & 6-27-HATpII & 359.5787 & 358.58486 & 0 & 406.1548 & 656.4678 & 1521.9564 \\
\hline 29 & $8-27$ & 62.9879 & 201.1050 & 0 & 0 & 0 & 1236.3037 \\
\hline 30 & 8-27-HATpII & 233.1508 & 280.9179 & 0 & 0 & 484.8050 & 1035.4888 \\
\hline 31 & $10-27$ & 17.1658 & 97.0037 & 0 & 0 & 0 & 849.8469 \\
\hline 32 & 10-27-HATpII & 111.6993 & 232.5512 & 0 & 0 & 0 & 953.7544 \\
\hline 33 & $12-27$ & 6.0098 & 50.0705 & 0 & 0 & 0 & 641.0816 \\
\hline 34 & 12-27-HATpII & 2.70154 & 41.9489 & 0 & 0 & 0 & 835.5134 \\
\hline 35 & $14-27$ & 1.49241 & 27.7714 & 0 & 0 & 0 & 605.9482 \\
\hline 36 & 14-27-НАТрII & 12.7723 & 74.91958 & 0 & 0 & 0 & 688.865 \\
\hline
\end{tabular}

problem. A number of experiments are performed with different design points. Simulation results show that when Inertia weight is lower than 0.5 and the number of iterations $=14$ the SCPSO performance is low, therefore we conclude that an inertia weight $=0.8$ is a good low threshold for this parameter. Consequently the high threshold should be modified up to find a suitable value to cope with more complex problem instances. Works [8] and [15] support the above observation, since authors show their exploration process to derive parameter values; however, they are not good for the present problem as its complexity increases.

On the other hand, HATp can provide better parameter values that improves the search ability of SCPSO to find a solution, enhancing its performance on 
An Experimental Study of Parameter Selection in Particle Swarm Optimization ...

Table 5. Tuning by analogy versus CALIBRA $P_{i}^{*}$ SCPSO results.

\begin{tabular}{|c|c|c|c|c|c|c|c|}
\hline & Design Point & Mean & $\begin{array}{l}\text { Standard } \\
\text { Deviation }\end{array}$ & Q1 & Median & Q3 & Maximum \\
\hline 1 & $4-15$ & 682.9017 & 181.5390 & 556.0250 & 673.705 & 794.495 & 1318.43 \\
\hline 2 & 4-15-HATpI & 659.3607 & 187.8980 & 527.1200 & 648.67 & 781.575 & 1303.3 \\
\hline 3 & $6-15$ & 635.4864 & 191.7692 & 504.0000 & 628.34 & 759.725 & 1364.72 \\
\hline 4 & 6-15-HATpI & 606.9634 & 221.6317 & 482.3050 & 621.485 & 745.45 & 1217.35 \\
\hline 5 & $8-15$ & 587.4071 & 214.8417 & 458.8550 & 590.33 & 707.28 & 1492.48 \\
\hline 6 & 8-15-HATpI & 145.8720 & 273.8555 & 0 & 0 & 0 & 1211.07 \\
\hline 7 & $10-15$ & 492.0968 & 223.2007 & 365.1100 & 501.86 & 627.47 & 1154.4 \\
\hline 8 & 10-15-HATpI & 532.4548 & 175.3387 & 413.64 & 522.845 & 629.95 & 1157.38 \\
\hline 9 & $12-15$ & 351.1337 & 227.8941 & 222.205 & 388.205 & 511.765 & 1124.26 \\
\hline 10 & 12-15-HATpI & 320.7400 & 231.2092 & 0 & 356.935 & 477.86 & 1016.81 \\
\hline 11 & $14-15$ & 220.2125 & 211.9554 & 0 & 251.25 & 376.045 & 1006.27 \\
\hline 12 & 14-15-HATpI & 367.0684 & 159.9957 & 271.54 & 348.345 & 455.86 & 938.5 \\
\hline 13 & $4-20$ & 654.1262 & 258.6153 & 518.245 & 667.765 & 823.59 & 1318.28 \\
\hline 14 & 4-20-HATpI & 704.7995 & 214.5675 & 571.155 & 698.325 & 847.28 & 1489.07 \\
\hline 15 & $6-20$ & 514.2564 & 315.9078 & 351.965 & 571.44 & 731.145 & 1388.26 \\
\hline 16 & 6-20-HATpI & 620.3161 & 229.7880 & 496.035 & 626.455 & 753.34 & 1471.96 \\
\hline 17 & $8-20$ & 364.8977 & 319.4826 & 0 & 427 & 621.625 & 1349.14 \\
\hline 18 & 8-20-HATpI & 467.7069 & 294.4225 & 331.835 & 517.33 & 673.465 & 1319.95 \\
\hline 19 & $10-20$ & 216.8342 & 285.9504 & 0 & 0 & 471.45 & 1248.8 \\
\hline 20 & 10-20-HATpI & 462.6305 & 225.4804 & 356.25 & 485.155 & 604.185 & 1056.78 \\
\hline 21 & $12-20$ & 107.2892 & 210.5406 & 0 & 0 & 0 & 1058.84 \\
\hline 22 & $12-20-\mathrm{HATpI}$ & 259.1259 & 264.9015 & 0 & 276.805 & 477.35 & 1088.38 \\
\hline 23 & $14-20$ & 40.8101 & 128.3257 & 0 & 0 & 0 & 868.99 \\
\hline 24 & 14-20-HATpI & 89.0967 & 173.724 & 0 & 0 & 0 & 986.5 \\
\hline 25 & $4-25$ & 373.3844 & 367.6206 & 0 & 431.3250 & 674.8550 & 1520.4800 \\
\hline 26 & 4-25-HATpI & 556.9658 & 317.0070 & 446.455 & 600.855 & 764.27 & 1378.4100 \\
\hline 27 & $6-25$ & 217.1393 & 322.6422 & 0 & 0 & 514.75 & 1307.2000 \\
\hline 28 & 6-25-HATpI & 459.6812 & 326.6541 & 0 & 532.61 & 698.265 & 1611.7400 \\
\hline 29 & $8-25$ & 97.4385 & 234.07921 & 0 & 0 & 0 & 1163.8100 \\
\hline 30 & 8-25-HATpI & 386.4427 & 300.07269 & 0 & 452.31 & 617.46 & 1214.9300 \\
\hline 31 & $10-25$ & 44.0648 & 157.0061 & 0 & 0 & 0 & 967.7300 \\
\hline 32 & 10-25-HATpI & 204.6662 & 285.2063 & 0 & 0 & 460.0800 & 1232.2100 \\
\hline 33 & $12-25$ & 12.6148 & 79.9636 & 0 & 0 & 0 & 814.1200 \\
\hline 34 & 12-25-HATpI & 43.7149 & 145.9721 & 0 & 0 & 0 & 990.7600 \\
\hline 35 & $14-25$ & 40.8101 & 128.3257 & 0 & 0 & 0 & 868.9900 \\
\hline 36 & 14-25-HATpI & 60.1733 & 158.4422 & 0 & 0 & 0 & 1075.4600 \\
\hline 37 & $4-30$ & 126.9177 & 282.2731 & 0 & 0 & 0 & 1264.15 \\
\hline 38 & 4-30-HATpI & 265.2456 & 360.2032 & 0 & 0 & 608.92 & 1453.76 \\
\hline 39 & $6-30$ & 55.7050 & 194.6109 & 0 & 0 & 0 & 1326 \\
\hline 40 & 6-30-HATpI & 41.4461 & 170.6329 & 0 & 0 & 0 & 1110.18 \\
\hline 41 & $8-30$ & 20.3758 & 115.4815 & 0 & 0 & 0 & 920.09 \\
\hline 42 & 8-30-HATpI & 145.8720 & 273.8555 & 0 & 0 & 0 & 1211.07 \\
\hline 43 & $10-30$ & 6.0608 & 59.3307 & 0 & 0 & 0 & 793.88 \\
\hline 44 & 10-30-HATpI & 14.0721 & 96.6330 & 0 & 0 & 0 & 1053.18 \\
\hline 45 & $12-30$ & 1.2604 & 23.8519 & 0 & 0 & 0 & 568.03 \\
\hline 46 & 12-30-HATpI & 2.8047 & 32.7819 & 0 & 0 & 0 & 618.89 \\
\hline 47 & $14-30$ & 0.4429 & 9.9828 & 0 & 0 & 0 & 250.14 \\
\hline 48 & 14-30-HATpI & 0 & 0 & $\mathbf{0}$ & 0 & 0 & 0 \\
\hline
\end{tabular}

resolving the spectrum sharing problem, than those parameters values suggested 
by TA and empirical methodology on some problem instances. This encourage us to analyse other regions using $H A T p$; in intention to find better $P^{*}$. Our interest is also to analyse another automated tuning procedures as ParamILS to gather information about how parameter values affect the SCPSO algorithm performance.

\section{References}

1. Eiben, A., Smit, S.: Parameter tuning for configuring and analyzing evolutionary algorithms. Swarm and Evolutionary Computation 1 (2011) $19-31$

2. Hutter, F., Hoos, H.H., Leyton-Brown, K., Murphy, K.P.: An experimental investigation of model-based parameter optimisation: Spo and beyond. In: Proceedings of the 11th Annual Conference on Genetic and Evolutionary Computation. GECCO '09, New York, NY, USA, ACM (2009) 271-278

3. Montero, E., Riff, M.C., Neveu, B.: A beginner's guide to tuning methods. Applied Soft Computing 17 (2014) 39 - 51

4. Kennedy, J., Eberhart, R.: Particle swarm optimization. In: Neural Networks, 1995. Proceedings., IEEE International Conference on. Volume 4. (1995) 1942-1948 vol.4

5. Parsopoulos, K., Vrahatis, M.: Particle Swarm Optimization and Intelligence: Advances and Applications. Premier reference source. Information Science Reference (2010)

6. Kennedy, J., Eberhart, R.C.: Swarm Intelligence. Morgan Kaufmann Publishers Inc., San Francisco, CA, USA (2001)

7. Kennedy, J., Eberhart, R.C.: A discrete binary version of the particle swarm algorithm. In: Systems, Man, and Cybernetics, 1997. Computational Cybernetics and Simulation., 1997 IEEE International Conference on. Volume 5., IEEE (1997) 4104-4108

8. Deep, K., Bansal, J.C.: A socio-cognitive particle swarm optimization for multidimensional knapsack problem. In: Proceedings of the 2008 First International Conference on Emerging Trends in Engineering and Technology. ICETET '08, Washington, DC, USA, IEEE Computer Society (2008) 355-360

9. Adenso-Diaz, B., Laguna, M.: Fine-tuning of algorithms using fractional experimental designs and local search. Oper. Res. 54 (2006) 99-114

10. Autar Kaw, E.E.K.: NUMERICAL METHODS WITH APPLICATIONS: Abridged. autarkaw.com (Licencia estndar de derechos de autor) (2011)

11. Coy, S.P., Golden, B.L., Runger, G.C., Wasil, E.A.: Using experimental design to find effective parameter settings for heuristics. Journal of Heuristics 7 (2001) $77-97$

12. Bartz-Beielstein, T.: How experimental algorithmics can benefit from mayo's extensions to neyman-pearson theory of testing. Synthese 163 (2008) 385-396

13. Dobslaw, F.: Recent development in automatic parameter tuning for metaheuristics. In: Proceedings of the 19th Annual Conference of Doctoral Students - WDS 2010. (2010)

14. MartíNez-Vargas, A., Andrade, A.G.: Comparing particle swarm optimization variants for a cognitive radio network. Appl. Soft Comput. 13 (2013) 1222-1234

15. Shi, Y., Eberhart, R.C.: Parameter selection in particle swarm optimization. In: Proceedings of the 7th International Conference on Evolutionary Programming VII. EP '98, London, UK, UK, Springer-Verlag (1998) 591-600 